\title{
Universal Sentence Representation Learning with Conditional Masked Language Model
}

\author{
Ziyi Yang ${ }^{1 *}$, Yinfei Yang ${ }^{2}$, Daniel Cer ${ }^{2}$, Jax Law $^{2}$, Eric Darve ${ }^{1}$ \\ ${ }^{1}$ Stanford University \\ \{ziyi.yang, darve\}@stanford.edu \\ ${ }^{2}$ Google Research \\ \{yinfeiy, cer, jaxlaw\}@google.com
}

\begin{abstract}
This paper presents a novel training method, Conditional Masked Language Modeling (CMLM), to effectively learn sentence representations on large scale unlabeled corpora. CMLM integrates sentence representation learning into MLM training by conditioning on the encoded vectors of adjacent sentences. Our English CMLM model achieves state-ofthe-art performance on SentEval (Conneau and Kiela, 2018), even outperforming models learned using supervised signals. As a fully unsupervised learning method, CMLM can be conveniently extended to a broad range of languages and domains. We find that a multilingual CMLM model co-trained with bitext retrieval (BR) and natural language inference (NLI) tasks outperforms the previous state-of-the-art multilingual models by a large margin, e.g. $10 \%$ improvement upon baseline models on cross-lingual semantic search. We explore the same language bias of the learned representations, and propose a simple, post-training and model agnostic approach to remove the language identifying information from the representation while still retaining sentence semantics.
\end{abstract}

\section{Introduction}

Sentence embeddings map sentences into a vector space. The vectors capture rich semantic information that can be used to measure semantic textual similarity (STS) between sentences or train classifiers for a broad range of downstream tasks (Conneau et al., 2017; Subramanian et al., 2018; Logeswaran and Lee, 2018; Cer et al., 2018; Reimers and Gurevych, 2019; Yang et al., 2019a,e). Stateof-the-art models are usually trained on supervised tasks such as natural language inference (Conneau et al., 2017), or with semi-structured data like question-answer pairs (Cer et al., 2018) and translation pairs (Subramanian et al., 2018; Yang et al.,

\footnotetext{
${ }^{*}$ Work done during internship at Google Research.
}

2019a). However, labeled and semi-structured data are difficult and expensive to obtain, making it hard to cover many domains and languages. Conversely, recent efforts to improve language models include the development of masked language model (MLM) pre-training from large scale unlabeled corpora (Devlin et al., 2019; Lan et al., 2020; Liu et al., 2019). While internal MLM model representations are helpful when fine-tuning on downstream tasks, they do not directly produce good sentence representations, without further supervised (Reimers and Gurevych, 2019) or semi-structured (Feng et al., 2020) fine-tuning.

In this paper, we explore an unsupervised approach, called Conditional Masked Language Modeling (CMLM), to effectively learn sentence representations from large scale unlabeled corpora. The CMLM model architecture is illustrated in Fig. 1, which integrates sentence representation learning into MLM training by conditioning on sentence level representations produced by adjacent sentences. The model therefore needs to learn effective sentence representations in order to perform good MLM. Since CMLM is fully unsupervised, it can be easily extended to new languages. We explore CMLM for both English and multilingual sentence embeddings for 100+ languages. Our English CMLM model achieves state-of-the-art performance on SentEval (Conneau and Kiela, 2018), even outperforming models learned using (semi)supervised signals. Moreover, models training on the English Amazon review data using our multilingual vectors exhibit strong multilingual transfer performance on translations of the Amazon review evaluation data to French, German and Japanese, outperforming existing multilingual sentence embedding models by $>5 \%$ for non-English languages and by $>2 \%$ on English.

We further extend the multilingual CMLM to cotrain with parallel text (bitext) retrieval task, and finetune with cross-lingual natural language infer- 


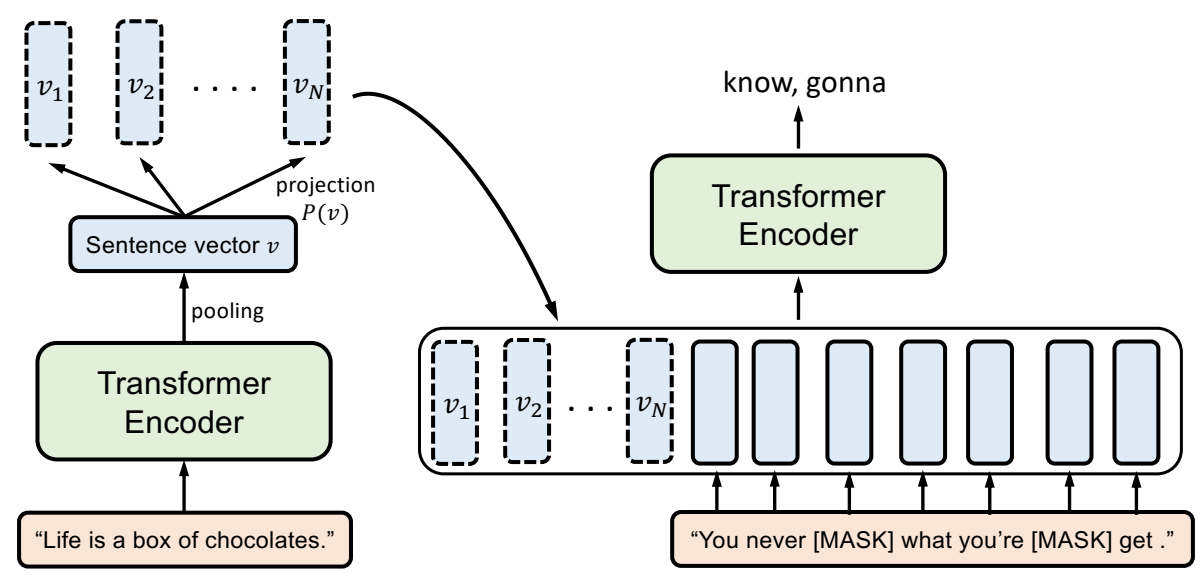

Figure 1: The architecture of Conditional Masked Language Modeling (CMLM).

ence (NLI) data, inspired by the success of prior work on multitask sentence representation learning (Subramanian et al., 2018; Yang et al., 2019a; Reimers and Gurevych, 2020) and NLI learning (Conneau et al., 2017; Reimers and Gurevych, 2019). We achieve performance $3.6 \%$ better than the previous state-of-the-art multilingual sentence representation model (see details in Section 4.2). On cross-lingual semantic search task, our model outperforms baseline models by $10 \%$ on average over 36 languages. Language agnostic representations require semantically similar cross-lingual pairs to be closer in representation space than unrelated same-language pairs (Roy et al., 2020). While we find our original sentence embeddings do have a bias for same language sentences, we discover that removing the first few principal components of the embeddings eliminates the self language bias.

The rest of the paper is organized as follows. Section 2 describes the architecture for CMLM unsupervised learning. In Section 3 we present CMLM trained on English data and evaluation results on SentEval. In Section 4 we apply CMLM to learn sentence multilingual sentence representations. Multitask training strategies on how to effectively combining CMLM, bitext retrieval and crosslingual NLI finetuning are explored. In Section 5, we investigate self language bias in multilingual representations and propose a simple but effective approach to eliminate it. The pre-trained models are released at https://tfhub.dev/s?q= universal-sentence-encoder-cmlm.

\section{Conditional Masked Language Modeling}

We introduce Conditional Masked Language Modeling (CMLM) as a novel architecture for combin- ing next sentence prediction with MLM training. By "conditional", we mean the MLM task for one sentence depends on the encoded sentence level representation of the adjacent sentence. This builds on prior work on next sentence prediction that has been widely used for learning sentence level representations (Kiros et al., 2015; Logeswaran and Lee, 2018; Cer et al., 2018; Yang et al., 2019a), but has thus far produced poor quality sentence embeddings within BERT based models using MLM loss (Reimers and Gurevych, 2019).

While existing MLMs like BERT include next sentence prediction tasks, they do so without any inductive bias to try to encode the meaning of a sentence within a single embedding vector. We introduce a strong inductive bias for learning sentence embeddings by structuring the task as follows. Given a pair of ordered sentences, the first sentence is fed to an encoder that produces a sentence level embedding. The embedding is then provided to an encoder that conditions on the sentence embedding in order to better perform MLM prediction over the second sentence. This is notably similar to Skip-Thought (Kiros et al., 2015), but replaces the generation of the complete second sentence with the MLM denoising objective. It is also similar to T5's MLM inspired unsupervised encode-decoder objective (Raffel et al., 2019), with the second encoder acting as a sort of decoder given the representation produced for the first sentence. Our method critically differs from T5's in that a sentence embedding bottleneck is used to pass information between two model components and in that the task involves denoising a second sentence when conditioning on the first rather than denoising a single text stream.

Fig. 1 illustrates the architecture of our model. 
The first sentence $s_{1}$ is tokenized and input to a transformer encoder and a sentence vector $\boldsymbol{v} \in \mathbb{R}^{d}$ is computed from the sequence outputs by average pooling. ${ }^{1}$ The sentence vector $v$ is then projected into $N$ spaces with one of the projections being the identity mapping, i.e. $\boldsymbol{v}_{p}=P(\boldsymbol{v}) \in \mathbb{R}^{d \times N}$. Here we use a three-layer MLP as the projection $P(\cdot)$. Details of $P(\cdot)$ are available in the supplementary material. One motivation for the projections of $s_{1}$ is that MLM of $s_{2}$ then can attend to various representations of $s_{1}$ instead of only 1 . In Section 5.1, we explore various different configurations of CMLM, including the number of projection spaces $N$.

The second sentence $s_{2}$ is then masked following the procedure described in the original BERT paper, including random replacement and the use of unchanged tokens. The second encoder shares the same weights with the encoder used to embed $s_{1}{ }^{2}$. Tokens in the masked $s_{2}$ are first converted into token vectors. The masked language modeling of $s_{2}$ depends on $s_{1}$ such that the process involves cross-attention between $s_{2}$ token vectors and $\boldsymbol{v}_{p}$. In practice, this is implemented by concatenating token embeddings of $s_{2}$ with $\boldsymbol{v}_{p}{ }^{3}$. Other implementations are also experimented (see Section 5.1) and we empirically find concatenation works the best. The concatenated representations are then provided to the transformer encoder to predict the masked tokens in $s_{2}$.

At inference time, we keep the first encoding module and discard the subsequent MLM prediction. Similar to skip-thought, CMLM trains the encoder to produce sentence embeddings useful for predicting material in the adjunct sentences. CMLM adapts this existing idea to MLM training. Appending multiple projections performs well due to fine-grained attention between tokens and the different views of the sentence embeddings. Note that CMLM differs from SkipThought in the following aspects: (a) SkipThought relies on an extra decoder network while CMLM only has the encoder. (b) SkipThought predicts the entire sentence while CMLM predicts masked tokens only so the

\footnotetext{
${ }^{1}$ One can equivalently choose other pooling methods, such as max pooling or use the vector output corresponding to a special token position such as the [CLS] token.

${ }^{2}$ The dual-encoder sharing encoder weights for different inputs can be also referred as "siamese encoder"

${ }^{3}$ Representation concatenation has been used in previous work for enabling cross attention between global vectors and local token embeddings to help the representations learning of long/structured inputs (Ainslie et al., 2020; Manzil Zaheer, 2020).
}

predictions can be done in parallel. These two differences make CMLM more efficient to train than SkipThought.

\section{Learning English Sentence Representations with CMLM}

For training English sentence encoders with CMLM, we use three Common Crawl dumps. The data are filtered by a classifier which detects whether a sentence belongs to the main content of the web page or not. We use WordPiece tokenization and the vocabulary is the same as public English uncased BERT. In order to enable the model to learn bidirectional information, for two consecutive sequences $s_{1}$ and $s_{2}$, we swap their order for $50 \%$ of the time. This order-swapping process echos with the preceding and succeeding sentences prediction in Skip-Thought (Kiros et al., 2015). The length of $s_{1}$ and $s_{2}$ are set to be 256 tokens (the maximum length). The number of masked tokens in $s_{2}$ are 80 (31.3\%), moderately higher than classical BERT. This change in the ratio of masked tokens is to make the task more challenging, due to the fact that in CMLM, language modeling has access to extra information from adjacent sentences. We train with batch size of 2048 for 1 million steps. The optimizer is LAMB (You et al., 2020) with learning rate of $10^{-3}, \beta_{1}=0.9, \beta_{2}=0.999$, warm-up in the first 10,000 steps and linear decay afterwards. We explore two transformer configurations same as in the original BERT paper, i.e., base and large. The number of projections $N$ is 15 by experimenting with multiple choices.

\subsection{Evaluation}

We evaluate the sentence representations on the following tasks: (1) classification: MR (movie reviews Pang and Lee (2005)), binary SST (sentiment analysis, Socher et al. (2013)), TREC (questiontype, Voorhees and Tice (2000)), CR (product reviews, Hu and Liu (2004)), SUBJ (subjectivity/objectivity, Pang and Lee (2004)). (2) Entailment: SICK dataset for entailment (SICK-E, Marelli et al. (2014)). The evaluation is done using SentEval (Conneau and Kiela, 2018) which is a prevailing evaluation toolkit for sentence embeddings. The classifier for the downstream is logistic regression. For each task, the encoder and embeddings are fixed and only downstream neural structures are trained.

The baseline sentence embedding models in- 
clude SkipThought (Kiros et al., 2015), InferSent (Conneau et al., 2017), USE (Cer et al., 2018), QuickThought (Logeswaran and Lee, 2018) and English BERT using standard pre-trained models from TensorFlow Hub website (Devlin et al., 2019), XLNet (Yang et al., 2019d), RoBERTa (Liu et al., 2019), SBert (Reimers and Gurevych, 2019). To evaluate the possible improvements coming from training data and processes, we train standard BERT models (English BERT base/large (CC)) on the same Common Crawl Corpora that CMLM is trained on. Similarly, we also train QuickThought, a competitive unsupervised sentence representations learning model, on the same Common Crawl Corpora (denoted as "QuickThought (CC)"). To further address the possible advantage from using Transformer encoder, we use a Transformer encoder as the sentence encoder in QuickThought (CC). The representations for BERT are computed by averaging the sequence outputs (we also explore options including [CLS] vector and max pooling and the results are available in the appendix).

\subsection{Results}

Evaluation results are presented in Table 1. The numbers are averaged over 5 runs and the performance variances are provided in the appendix. CMLM outperforms existing models overall, besting MLM (both English BERT and English BERT (CC)) using both base and large configurations. The closest competing model is SBERT, which uses supervised NLI data rather than a purely unsupervised approach. Interestingly, CMLM outperforms SBERT on the SICK-E NLI task even the later model is trained with a NLI task. We also evaluate on Semantic Textual Similarity (STS) datasets. As shown in Table 2, CMLM exhibits competitive performance compared with BERT and GloVe. One interesting observation is that CMLM base significantly outperforms other baselines (including CMLM large) on the STS Benchmark dataset.

\section{Learning Multilingual Sentence Representations with CMLM}

As a fully unsupervised method, CMLM can be conveniently extended to multilingual modeling even for less well resourced languages. Learning good multilingual sentence representations is more challenging than monolingual ones, especially when attempting to capture the semantic alignment between different languages. As CMLM does not explicitly address cross-lingual alignment, we explore several modeling approaches besides CMLM: (1) Co-training CMLM with a bitext retrieval task; (2) Fine-tuning with cross-lingual NLI data.

\subsection{Multilingual CMLM}

We follow the same configuration used to learn English sentence representations with CMLM, but extend the training data to include more languages. Results below will show that CMLM again exhibits competitive performance as a general technique to learn from large scale unlabeled corpora.

\subsection{Multitask Training with CMLM and Bitext Retrieval}

Besides the monolingual pretraining data, we collect a dataset of bilingual translation pairs $\left\{\left(s_{i}, t_{i}\right)\right\}$ using a bitext mining system (Feng et al., 2020). The source sentences $\left\{s_{i}\right\}$ are in English and the target sentences $\left\{t_{i}\right\}$ covers over 100 languages. We build a retrieval task with the translation parallel data, identifying the corresponding translation of the input sentence from candidates in the same batch. Concretely, incorporating Additive Margin Softmax (Yang et al., 2019b), we compute the bitext retrieval loss $\mathcal{L}_{b r}^{s}$ for the source sentences as:

$$
\mathcal{L}_{b r}^{s}=-\frac{1}{B} \sum_{i=1}^{B} \frac{e^{\phi\left(s_{i}, t_{i}\right)-m}}{e^{\phi\left(s_{i}, t_{i}\right)-m}+\sum_{j=1, j \neq i}^{B} e^{\phi\left(s_{i}, t_{j}\right)}}
$$

Above $\phi\left(s_{i}, t_{j}\right)$ denotes the the inner products of sentence vectors of $s_{i}$ and $t_{j}$ (embedded by the transformer encoder); $m$ and $B$ denotes the additive margin and the batch size respectively. Note the way to generate sentence embeddings is the same as in CMLM. We can compute the bitext retrieval loss for the target sentences $\mathcal{L}_{b r}^{t}$ by normalizing over source sentences, rather than target sentences, in the denominator. ${ }^{4}$ The final bitext retrieval loss $\mathcal{L}_{b r}$ is given as $\mathcal{L}_{b r}=\mathcal{L}_{b r}^{s}+\mathcal{L}_{b r}^{t}$.

There are several ways to incorporate the monolingual CMLM task and bitext retrieval (BR). We explore the following multistage and multitask pretraining strategies:

S1. CMLM+BR: Train with CMLM and BR from the start;

S2. CMLM $\rightarrow$ BR: Train with CMLM in the first stage and then train with on BR;

\footnotetext{
${ }^{4}$ i.e., by swapping the $i$ and $j$ subscripts in the last term of the denominator.
} 


\begin{tabular}{|c|c|c|c|c|c|c|c|c|c|c|}
\hline Model & MR & $\mathbf{C R}$ & SUBJ & MPQA & SST & TREC & MRPC & SICK-E & SICK-R & Avg. \\
\hline SkipThought & 76.5 & 80.1 & 93.6 & 87.1 & 82.0 & 92.2 & 73.0 & 82.3 & 85.8 & 83.8 \\
\hline InferSent & 81.6 & 86.5 & 92.5 & 90.4 & 84.2 & 88.2 & 75.8 & 84.3 & 86.4 & 85.5 \\
\hline USE & 80.1 & 85.2 & 94.0 & 86.7 & 86.4 & 93.2 & 70.1 & 82.4 & 85.9 & 84.9 \\
\hline QuickThought (CC) & 75.7 & 81.9 & 94.3 & 84.7 & 79.7 & 83.0 & 70.4 & 75.0 & 78.5 & 80.4 \\
\hline XLNet & 83.6 & 82.1 & 90.8 & 89.0 & 89.0 & 90.4 & 70.1 & 82.1 & 78.4 & 83.9 \\
\hline \multicolumn{11}{|c|}{ BERT-based models } \\
\hline English BERT base & 81.6 & 87.4 & 95.2 & 87.8 & 85.8 & 90.6 & 71.1 & 79.3 & 80.5 & 84.3 \\
\hline English BERT base (CC) & 82.5 & 88.5 & 95.6 & 87.3 & 88.0 & 91.4 & 72.0 & 79.3 & 79.0 & 84.6 \\
\hline SBERT (NLI, base) & 83.6 & 89.4 & 94.4 & 89.9 & 88.9 & 89.6 & 76.0 & 79.9 & 80.6 & 85.8 \\
\hline CMLM base (ours) & 83.6 & 89.9 & 96.2 & 89.3 & 88.5 & 91.0 & 69.7 & 82.3 & 83.4 & 86.0 \\
\hline English BERT large & 84.3 & 88.9 & 95.7 & 86.8 & 88.9 & 91.4 & 71.8 & 75.7 & 77.0 & 84.5 \\
\hline English BERT large (CC) & 85.4 & 89.0 & 95.7 & 86.9 & 90.5 & 91.2 & 75.5 & 74.3 & 77.0 & 85.0 \\
\hline RoBERTa (large) & 85.2 & 90.6 & 97.0 & 90.0 & 89.5 & 93.6 & 74.2 & 75.1 & 78.9 & 86.0 \\
\hline SBERT (NLI, large) & 84.8 & 90.0 & 94.5 & 90.3 & 90.7 & 87.4 & 76.0 & 74.9 & 84.2 & 85.9 \\
\hline CMLM large (ours) & 85.6 & 89.1 & 96.6 & 89.3 & 91.4 & 92.4 & 70.0 & 82.2 & 84.5 & 86.8 \\
\hline
\end{tabular}

Table 1: Transfer learning test set results on SentEval for English models. Baseline models include BERT-based (BERT, RoBERTA and SBERT) and non-BERT models (XLNet, SkipThought, InferSent and USE).

\begin{tabular}{l|cccccccc}
\hline Model & STS12 & STS13 & STS14 & STS15 & STS16 & STSB & SICK-R & Avg. \\
\hline Avg. GloVe embeddings & 55.14 & $\mathbf{7 0 . 6 6}$ & 59.73 & 68.25 & 63.66 & 58.02 & 53.76 & 61.32 \\
BERT Mean embeddings & 38.78 & 57.98 & 57.98 & 63.15 & 61.06 & 46.35 & 58.40 & 54.81 \\
BERT CLS-vector & 20.16 & 30.01 & 20.09 & 36.88 & 38.08 & 16.50 & 42.63 & 29.19 \\
CMLM base (ours) & 58.20 & 61.07 & 61.67 & 73.32 & 74.88 & $\mathbf{7 6 . 6 0}$ & 64.80 & $\mathbf{6 7 . 2 2}$ \\
CMLM large (ours) & $\mathbf{5 9 . 0 2}$ & 61.68 & $\mathbf{6 2 . 8 0}$ & $\mathbf{7 4 . 1 6}$ & $\mathbf{7 5 . 6 4}$ & 69.39 & $\mathbf{6 6 . 5 6}$ & 67.03 \\
\hline
\end{tabular}

Table 2: Spearman rank correlation on Semantic Textual Similarity (STS) datasets. SICK-R is zero-shot evaluation by directly computing the cosine similarity of sentence embeddings, without training the task-specific neural network.

S3. CMLM $\rightarrow$ CMLM+BR: Train with only CMLM in the first stage and then with both tasks.

When training with both CMLM and BR, the optimization loss is a weighted sum of the language modeling and the retrieval loss $\mathcal{L}_{b r}$, i.e. $\mathcal{L}=\mathcal{L}_{C M L M}+\alpha \mathcal{L}_{b r}$. We empirically find $\alpha=0.2$ works well. As shown in Table 4, S3 is found to be the most effective. Unless otherwise denoted, our models trained with CMLM and BR follow S3. We also discover that given a pre-trained transformer encoder, e.g. mBERT, we can improve the quality of sentence representations by finetuning the transformer encoder with CMLM and BR. As shown in Table 4, the improvements of f-mBERT (finetuned mBERT) upon mBERT are significant.

\subsection{Finetuning with Cross-lingual Natural Language Inference}

Finetuning with NLI data has proved to be an effective method to improve the quality of embeddings for English models. We propose to leverage cross-lingual NLI finetuning in multilingual representations. Given a premise sentence $\boldsymbol{u}$ in language $l_{1}$ and a hypothesis sentence $\boldsymbol{v}$ in language $l_{2}$, we train a 3-way classifier on the concatenation of $[\boldsymbol{u}, \boldsymbol{v},|\boldsymbol{u}-\boldsymbol{v}|, \boldsymbol{u} * \boldsymbol{v}]$. Weights of transformer encoders are also updated in the finetuning process. Different from previous work also using multilingual NLI data (Yang et al., 2019a), the premise $\boldsymbol{u}$ and hypothesis $v$ are in different languages. The cross-lingual NLI data are generated by translating Multi-Genre NLI Corpus (Williams et al., 2018) into 14 languages using Google Translate API.

\subsection{Configurations}

Monolingual training data for CMLM are generated from 3 versions of Common Crawl data in 113 languages. The data cleaning and filtering is the same as the English-only ones. A new cased vocabulary is built from the all data sources using the WordPiece vocabulary generation library from Tensorflow Text. The language smoothing exponent from the vocab generation tool is set to 0.3 , as the distribution of data size for each language is imbalanced. The final vocabulary size is 501,153 . The number of projections $N$ is set to be 15 , the batch size $B$ is 2048 and the positive margin is 0.3 . 


\begin{tabular}{lcccccccccccccccc}
\hline Model & ar & bg & de & el & en & es & fr & hi & ru & sw & th & tr & ur & vi & zh & Avg. \\
\hline mBERT & 76.3 & 76.1 & 77.7 & 76.1 & 80.1 & 78.5 & 78.7 & 75.6 & 77.3 & 70.5 & 73.6 & 75.7 & 74.2 & 78.8 & 78.7 & 76.5 \\
MLM (CC) & 79.2 & 79.1 & 81.7 & 79.9 & 84.4 & 82.1 & 82.2 & 79.2 & 81.2 & 70.3 & 76.9 & 79.0 & 74.3 & 81.3 & 81.0 & 79.4 \\
XLM-R & 78.1 & 78.0 & 76.2 & 78.2 & 82.8 & 81.2 & 80.4 & 77.2 & 80.2 & 71.0 & 77.5 & 79.7 & 76.7 & 80.3 & 80.8 & 78.5 \\
CMLM & $\mathbf{8 0 . 6}$ & $\mathbf{8 1 . 2}$ & $\mathbf{8 2 . 6}$ & $\mathbf{8 1 . 4}$ & $\mathbf{8 5 . 0}$ & $\mathbf{8 2 . 3}$ & $\mathbf{8 3 . 4}$ & $\mathbf{8 0 . 0}$ & $\mathbf{8 2 . 3}$ & $\mathbf{7 6 . 2}$ & $\mathbf{7 8 . 8}$ & $\mathbf{8 1 . 0}$ & $\mathbf{7 8 . 5}$ & $\mathbf{8 1 . 6}$ & $\mathbf{8 1 . 7}$ & $\mathbf{8 1 . 2}$ \\
\hline
\end{tabular}

Table 3: Performance (accuracy) of multilingual models trained with monolingual data on XEVAL. Highest numbers are highlighted in bold.

\begin{tabular}{lcccccccccccccccc}
\hline Model & ar & bg & de & el & en & es & fr & hi & ru & sw & th & tr & ur & vi & zh & Avg. \\
\hline LASER & 82.1 & 81.2 & 81.7 & 78.1 & 82.3 & 81.0 & 80.8 & 78.9 & 82.2 & 75.8 & 80.3 & 81.8 & 77.2 & 81.6 & 82.1 & 80.4 \\
mUSE & 80.4 & - & 82.2 & - & 83.3 & 82.7 & 82.4 & - & 82.3 & - & 81.6 & 80.3 & - & - & 82.0 & 81.9 \\
S1 & 78.3 & 78.9 & 79.3 & 78.1 & 81.0 & 78.7 & 79.5 & 78.0 & 79.0 & 76.6 & 77.8 & 78.6 & 77.7 & 79.0 & 78.6 & 78.6 \\
S2 & 81.3 & 81.0 & 83.0 & 81.4 & 85.6 & 83.0 & 83.6 & 80.4 & 82.3 & 77.6 & 80.1 & 81.0 & 79.8 & 82.4 & 82.3 & 81.6 \\
S3 & 82.6 & 83.0 & 84.0 & 81.8 & 85.8 & 84.2 & 84.6 & 81.7 & 84.0 & 79.3 & 81.2 & 82.7 & 81.2 & 83.0 & 83.0 & 82.8 \\
S3+NLI & $\mathbf{8 4 . 2}$ & $\mathbf{8 3 . 7}$ & $\mathbf{8 5 . 0}$ & $\mathbf{8 3 . 4}$ & $\mathbf{8 7 . 0}$ & $\mathbf{8 5 . 9}$ & $\mathbf{8 5 . 8}$ & $\mathbf{8 3 . 0}$ & $\mathbf{8 5 . 6}$ & $\mathbf{7 9 . 6}$ & $\mathbf{8 3 . 0}$ & $\mathbf{8 4 . 2}$ & $\mathbf{8 1 . 2}$ & $\mathbf{8 4 . 2}$ & $\mathbf{8 4 . 4}$ & $\mathbf{8 4 . 0}$ \\
\hline mBERT & 76.3 & 76.1 & 77.7 & 76.1 & 80.1 & 78.5 & 78.7 & 75.6 & 77.3 & 70.5 & 73.6 & 75.7 & 74.2 & 78.8 & 78.7 & 76.5 \\
f-mBERT & 77.2 & 78.5 & 79.7 & 76.7 & 81.4 & 80.0 & 80.3 & 77.2 & 79.1 & 73.3 & 76.1 & 77.1 & 76.9 & 79.8 & 80.4 & 78.3 \\
\hline
\end{tabular}

Table 4: Performance (accuracy) of models trained with cross-lingual data on XEVAL. We test with multiple strategies for multitask pretraining: [S1]: CMLM $\rightarrow$ BR; [S2]: CMLM+BR; [S3]: CMLM $\rightarrow$ CMLM+BR. [f-mBERT] denotes finetuning mBERT with CMLM and BR.

For CMLM only pretraining, the number of steps is 2 million. In multitask learning, for $\mathrm{S} 1$ and $\mathrm{S} 3$, the first stage is of 1.5 million and the second stage is of 1 million steps; for S2, number of training steps is 2 million. The transformer encoder uses the BERT base configuration. Initial learning rate and optimizer chosen are the same as the English models. Motivations for choosing such configurations, training details and potential limitations of CMLM are discussed in the appendix.

\subsection{Evaluations}

\subsubsection{XEVAL: Multilingual Benchmarks for Sentence Representations Evaluation}

Evaluations in previous multilingual literature focused on the cross-lingual transfer learning ability from English to other languages. However, this evaluation protocol that treats English as the "anchor" does not equally assess the quality of nonEnglish sentence representations with English ones. To address the issue, we prepare a new benchmark for multilingual sentence vectors, XEVAL, by translating SentEval (English) to other 14 languages with Google Translate API. The reliability of XEVAL is discussed in the appendix.

Results of models trained with monolingual data are shown in Table 3. Baseline models include mBERT (Devlin et al., 2019), XLM-R (Ruder et al., 2019) and a transformer encoder trained with MLM on the same Common Crawl data (MLM(CC), again this is to control the effects of training data).
The method to produce sentence representations for mBERT and XLM-R is chosen to be average pooling after exploring options including [CLS] representations and max pooling. The multilingual model CMLM trained on monolingual data outperform all baselines in all 15 languages.

Results of models trained with cross-lingual data are presented in Table 4. Baseline models for comparison include LASER (Artetxe and Schwenk (2019), trained with parallel data) and multilingual USE ((Yang et al., 2019a), trained with crosslingual NLI. Note it only supports 16 languages). Our model (S3) outperforms LASER in all 15 languages. Notably, finetuning with NLI in the crosslingual way produces significant improvement (S3 + NLI v.s. S3). Multitask learning with CMLM and BR can also be used to increase the performance of pretrained encoders, e.g. mBERT. mBERT trained with CMLM and BR (f-mBERT) has a significant improvement upon mBERT.

\subsubsection{Amazon Reviews}

We conduct a zero-shot transfer learning evaluation on Amazon reviews dataset (Prettenhofer and Stein, 2010). Following Chidambaram et al. (2019), the original dataset is converted to a classification benchmark by treating reviews with strictly more than 3 stars as positive and negative otherwise. We split 6000 English reviews in the original training set into $90 \%$ for training and $10 \%$ for development. The two-way classifier, upon the concatena- 


\begin{tabular}{|c|c|c|c|c|c|c|c|c|c|c|c|c|c|c|c|c|c|c|c|}
\hline Lang. & af & ar & bg & bn & de & el & es & et & $\mathrm{eu}$ & fa & fi & $\mathrm{fr}$ & he & hi & hu & id & it & ja & \\
\hline mBERT & 42.7 & 25.8 & 49.3 & 17 & 77.2 & 29.8 & 68.7 & 29.3 & 25.5 & 46.1 & 39 & 66.3 & 41.9 & 34.8 & 38.7 & 54.6 & 58.4 & 42 & \\
\hline MLM (CC) & 60.5 & 51.4 & 74.8 & 45 & 89.3 & 68.3 & 81.8 & 56.8 & 59.5 & 81.3 & 76.6 & 82.6 & 72.2 & 76.2 & 68.4 & 82.6 & 72.8 & 65.7 & \\
\hline XLM & 43.2 & 18.2 & 40 & 13.5 & 66.2 & 25.6 & 58.4 & 24.8 & 17.1 & 32.2 & 32.2 & 54.5 & 32.1 & 26.5 & 30.1 & 45.9 & 56.5 & 40 & \\
\hline XLM-R & 58.2 & 47.5 & 71.6 & 43 & 88.8 & 61.8 & 75.7 & 52.2 & 35.8 & 70.5 & 71.6 & 73.7 & 66.4 & 72.2 & 65.4 & 77 & 68.3 & 60.6 & \\
\hline LASER & 89.4 & 91.9 & 95.0 & 89.6 & 99.0 & 94.9 & 98.0 & 96.7 & 94.6 & 71.6 & 96.3 & 95.6 & 92.1 & 94.7 & 96.0 & 94.5 & 95.4 & 95.3 & \\
\hline CMLM & 62.0 & 53.2 & 75.0 & 45.1 & 89.9 & 69.9 & 82.7 & 59.2 & 61.6 & 83.7 & 77.1 & 83.5 & 73.1 & 76.7 & 70.3 & 83.0 & 73.5 & 67.2 & \\
\hline CMLM+BR & 96.3 & 90.6 & 95.4 & 91.2 & 97.7 & 95.4 & 98.1 & 95.6 & 92.0 & 95.6 & 95.9 & 96.1 & 92.8 & 97.6 & 96.5 & 95.6 & 94.2 & 95.6 & \\
\hline \multirow{2}{*}{$\mathrm{CMLM+BR+NLI}$} & 90.5 & 83.6 & 92.6 & 86.4 & 97.6 & 91.6 & 95.5 & 82.6 & 76.3 & 90.7 & 88.9 & 93.5 & 86.8 & 94.6 & 89.6 & 91.7 & 90.4 & 88.4 & \\
\hline & jv & ka & $\mathrm{kk}$ & ko & $\mathrm{ml}$ & $\mathrm{mr}$ & $\mathrm{nl}$ & $\mathrm{pt}$ & ru & sw & ta & te & th & $\mathrm{tl}$ & $\operatorname{tr}$ & ur & vi & $\mathrm{zh}$ & Mean \\
\hline mBERT & 17.6 & 20.5 & 27.1 & 38.5 & 19.8 & 20.9 & 68 & 69.9 & 61.2 & 11.5 & 14.3 & 16.2 & 13.7 & 16 & 34.8 & 31.6 & 62 & 71.6 & 38.7 \\
\hline $\operatorname{MLM}(\mathrm{CC})$ & 49.5 & 65.8 & 61.3 & 66.4 & 65.3 & 56.8 & 83.4 & 83.1 & 74.8 & 65.9 & 61.3 & 68.5 & 70.0 & 62.7 & 70.3 & 80.1 & 77.0 & 71.3 & 69.4 \\
\hline XLM & 22.4 & 22.9 & 17.9 & 25.5 & 20.1 & 13.9 & 59.6 & 63.9 & 44.8 & 12.6 & 20.2 & 12.4 & 31.8 & 14.8 & 26.2 & 18.1 & 47.1 & 42.2 & 32.6 \\
\hline XLM-R & 14.1 & 52.1 & 48.5 & 61.4 & 65.4 & 56.8 & 80.8 & 82.2 & 74.1 & 20.3 & 26.4 & 35.9 & 29.4 & 36.7 & 65.7 & 24.3 & 74.7 & 68.3 & 57.3 \\
\hline LASER & 23.0 & 35.9 & 18.6 & 88.9 & 96.9 & 91.5 & 96.3 & 95.2 & 94.4 & 57.5 & 69.4 & 79.7 & 95.4 & 50.6 & 97.5 & 81.9 & 96.8 & 95.5 & 84.4 \\
\hline CMLM & 51.8 & 65.5 & 62.7 & 67.2 & 65.8 & 57.0 & 83.8 & 83.6 & 75.5 & 66.6 & 61.7 & 68.8 & 70.3 & 63.5 & 70.5 & 80.3 & 77.4 & 71.7 & 70.3 \\
\hline CMLM+BR & 83.4 & 94.9 & 88.6 & 92.4 & 98.9 & 94.5 & 97.3 & 95.3 & 94.9 & 87.0 & 91.2 & 97.9 & 96.6 & 95.3 & 98.6 & 94.4 & 97.5 & 95.6 & 94.7 \\
\hline $\mathrm{CMLM+BR+NLI}$ & 66.9 & 88.1 & 80.3 & 85.6 & 94.9 & 90.7 & 93.2 & 92.3 & 91.7 & 76.7 & 88.6 & 92.8 & 94.7 & 82.0 & 94.3 & 84.7 & 94.3 & 93.1 & 88.8 \\
\hline
\end{tabular}

Table 5: Tatoeba results (retrieval accuracy) for each language. Our model CMLM+BR achieves the best results on 30 out of 36 languages.

\begin{tabular}{lcccc}
\hline Models & English & French & German & Japanese \\
\hline Encoder parameters are & frozen & during finetuning & \\
\hline Eriguchi et al. (2018) & 83.2 & 81.3 & - & - \\
MTDE en-fr & 87.4 & 82.3 & - & - \\
MTDE en-de & 87.1 & - & 81.0 & - \\
mBERT & 80.0 & 73.1 & 70.4 & 71.7 \\
XLM-R & - & 85.3 & 81.5 & 82.5 \\
MLM (CC) & 84.6 & 84.9 & 84.3 & 82.1 \\
CMLM & 88.4 & 88.2 & 87.5 & $\mathbf{8 3 . 7}$ \\
CMLM+ BR & 88.3 & 87.2 & 86.4 & 83.2 \\
CMLM+ BR + NLI & $\mathbf{8 9 . 4}$ & $\mathbf{8 8 . 8}$ & $\mathbf{8 8 . 4}$ & 82.8 \\
\hline Encoder parameters are trained during finetuning & \\
\hline mBERT & 89.3 & 83.5 & 79.4 & 74.0 \\
MLM (CC) & 92.9 & 88.7 & 88.4 & 86.3 \\
CMLM & 93.4 & 92.4 & 92.1 & $\mathbf{8 8 . 6}$ \\
CMLM+ BR & 93.6 & $\mathbf{9 3 . 1}$ & 92.3 & 88.1 \\
CMLM+ BR + NLI & $\mathbf{9 3 . 7}$ & 92.4 & $\mathbf{9 3 . 5}$ & 86.8 \\
\hline
\end{tabular}

Table 6: Classification accuracy on the Amazon Reviews dataset.

tion of $[\boldsymbol{u}, \boldsymbol{v},|\boldsymbol{u}-\boldsymbol{v}|, \boldsymbol{u} * \boldsymbol{v}]$ (following works e.g. Reimers and Gurevych (2019)), is trained on the English training set and then evaluated on English, French, German and Japanese test sets (each has 6000 examples). The same multilingual encoder and classifier are used for all the evaluations. We also experiment with whether freezing the encoder weights or not during training. As presented in Table 6, CMLM alone has already outperformed baseline models, including Multi-task Dual-Encoder (MTDE, Chidambaram et al. (2019)), mBERT and XLM-R. Training with BR and cross-lingual NLI finetuning further boost the performance.

\subsection{Tatoeba: Semantic Search}

We test on Tatoeba dataset proposed in Artetxe and Schwenk (2019) to asses the ability of our models on capturing cross-lingual semantics. The task is to find the nearest neighbor for the query sentence in the other language. The experiments is conducted on the 36 languages as in XTREME (Hu et al., 2020). The evaluation metric is retrieval accuracy. Results are presented in Table 5. Our model CMLM+BR outperforms all baseline models in 30 out of 36 languages and has the highest average performance. One interesting observation is that finetuning with NLI actually undermines the model performance on semantic search, in contrary with the significant improvements from CMLM+BR to CMLM+BR+NLI on XEVAL (Table 4). We speculate this is because unlike semantic search, NLI inference is not a linear process. Finetuning with NLI is not expected to help the linear retrieval by nearest neighbor search.

\section{Analysis}

\subsection{Ablation Study}

We explore different configurations of CMLM, including the number of projection spaces $N$ (Table 7). Projecting the sentence vector into $N=15$ spaces produces highest overall performance. We also try a different CMLM architecture. Besides the concatenation with token embeddings of $s_{2}$ before input to the transformer encoder, the projected vectors are also concatenated with the sequence outputs of $s_{2}$ for the masked token prediction. This version of architecture is denoted as "skip" and the model performance is actually worse.

Note that the projected vector can also be used to produce the sentence representation $\boldsymbol{v}_{s}$, e.g. using the average of projected vectors $\boldsymbol{v}_{s}=\frac{1}{N} \sum_{i} \boldsymbol{v}_{p}^{(i)}$ as the sentence embeddings. Recall $\boldsymbol{v}_{p}^{(i)}$ is the $i$ th 


\begin{tabular}{l|cccccccccc}
\hline Model & MR & CR & SUBJ & MPQA & SST & TREC & MRPC & SICK-E & SICK-R & Avg. \\
\hline$N=1$ & 82.3 & 89.7 & 95.8 & 88.8 & 87.6 & 90.4 & $\mathbf{7 1 . 5}$ & 80.8 & 83.4 & 85.5 \\
$N=5$ & $\mathbf{8 3 . 7}$ & $\mathbf{9 0 . 0}$ & 95.5 & 89.0 & $\mathbf{8 9 . 4}$ & 86.6 & 69.5 & 79.3 & 81.7 & 85.0 \\
$N=10$ & 83.4 & 89.0 & 96.1 & 88.9 & 88.2 & 90.2 & 68.5 & 79.7 & 81.5 & 84.9 \\
$N=15$ & 83.6 & 89.9 & $\mathbf{9 6 . 2}$ & $\mathbf{8 9 . 3}$ & 88.5 & $\mathbf{9 1 . 0}$ & 69.7 & $\mathbf{8 2 . 3}$ & $\mathbf{8 3 . 4}$ & $\mathbf{8 6 . 0}$ \\
$N=20$ & 81.1 & 89.5 & 95.8 & 88.9 & 85.9 & 89.8 & 69.7 & 80.2 & 85.0 & 85.1 \\
skip & 80.3 & 86.8 & 94.5 & 87.5 & 84.9 & 86.0 & 69.2 & 72.8 & 74.7 & 81.9 \\
proj & 82.6 & 89.7 & 96.0 & 87.3 & 87.5 & 89.2 & 70.5 & 81.7 & 83.8 & 85.4 \\
\hline
\end{tabular}

Table 7: Ablation study of CMLM designs, including the number of projection spaces, architecture and sentence representations. The experiments are conducted on SentEval.

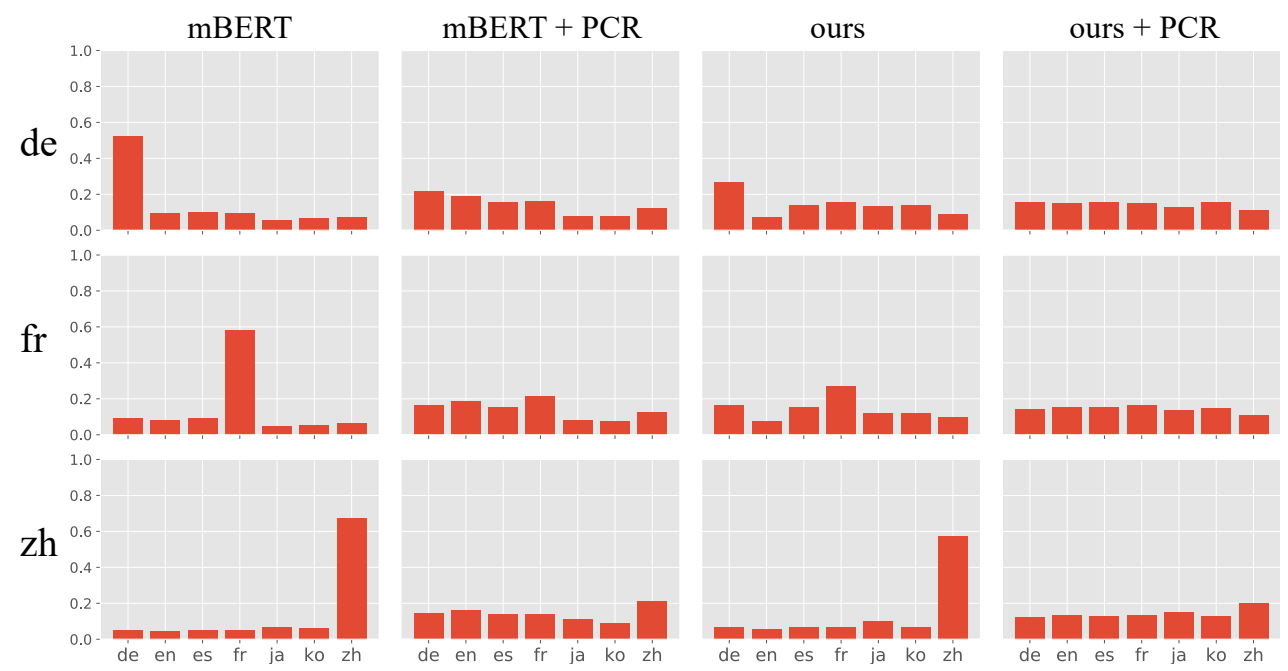

Figure 2: Language distribution of retrieved sentences. The histogram values represent the percentage of sentences retrieved in a language. The first and third columns are mBERT and our models. Our model already in general has a more uniform distribution than mBERT. The second and fourth columns are mBERT and our model with PCR.

projection. This version is denoted as "proj" in Table 7. Sentence representations produced in this way still yield competitive performance, which further confirm the effectiveness of the projection.

\begin{tabular}{l|cccccc}
\hline & fra & cmn & spa & deu & rus & ita \\
\hline mBERT & $\mathbf{6 0 . 2}$ & 60.2 & $\mathbf{6 2 . 8}$ & 65.9 & 53.8 & 55.7 \\
mBERT + PCR & 59.9 & $\mathbf{6 4 . 3}$ & 61.7 & $\mathbf{6 7 . 5}$ & $\mathbf{5 7 . 4}$ & $\mathbf{5 6 . 2}$ \\
\hline ours & $\mathbf{9 6 . 1}$ & 95.6 & 98.1 & 97.7 & 94.9 & $\mathbf{9 4 . 2}$ \\
ours + PCR & 95.5 & $\mathbf{9 6 . 0}$ & $\mathbf{9 8 . 2}$ & $\mathbf{9 7 . 9}$ & $\mathbf{9 5 . 1}$ & 94.1 \\
\hline \multirow{2}{*}{ mBERT } & tur & por & hun & jpn & nld & Avg. \\
mBERT + PCR & 32.4 & 62.4 & 31.9 & 39.0 & 56.2 & 52.8 \\
\hline ours & $\mathbf{3 3 . 3}$ & $\mathbf{6 4 . 4}$ & $\mathbf{3 6 . 5}$ & $\mathbf{4 2 . 3}$ & $\mathbf{6 1 . 1}$ & $\mathbf{5 4 . 8}$ \\
ours + PCR & 98.6 & 95.3 & 96.5 & $\mathbf{9 5 . 6}$ & $\mathbf{9 7 . 3}$ & 96.3 \\
\hline
\end{tabular}

Table 8: Average retrieval accuracy on 11 languages of multilingual representations model with and without PCR on Tatoeba dataset.

\subsection{Language Agnostic Properties}

Language Agnosticism has been a property of great interest for multilingual representations.
However, there has not been a qualitative measurement or rigid definition for this property. We propose that "language agnostic" refers to the property that sentences representations are neutral w.r.t their language information. E.g., two sentences with similar semantics should be close in embedding space whether they are of the same languages or not. To capture this intuition, we convert the PAWS$\mathrm{X}$ dataset (Yang et al., 2019c) to a retrieval task to measure the language agnostic property. Specifically, PAWS-X consists of English sentences and their translations in other six languages. Given a query, we inspect the language distribution of the retrieved sentences. The similarity between a query $\boldsymbol{v}_{l_{1}}$ in language $l_{1}$ and a candidate $\boldsymbol{v}_{l_{2}}$ in language $l_{2}$ is computed as the cosine similarity $\frac{\boldsymbol{v}_{l_{1}}^{T} \boldsymbol{v}_{l_{2}}}{\left\|\boldsymbol{v}_{l_{1}}\right\|_{2}\left\|\boldsymbol{v}_{l_{2}}\right\|_{2}}$. In Fig. 2, representations of mBERT have a strong self language bias, i.e. sentences in the language matching the query are dominant. In contrast, the bias is much weaker in our model, probably due to the cross-lingual retrieval pretraining. 

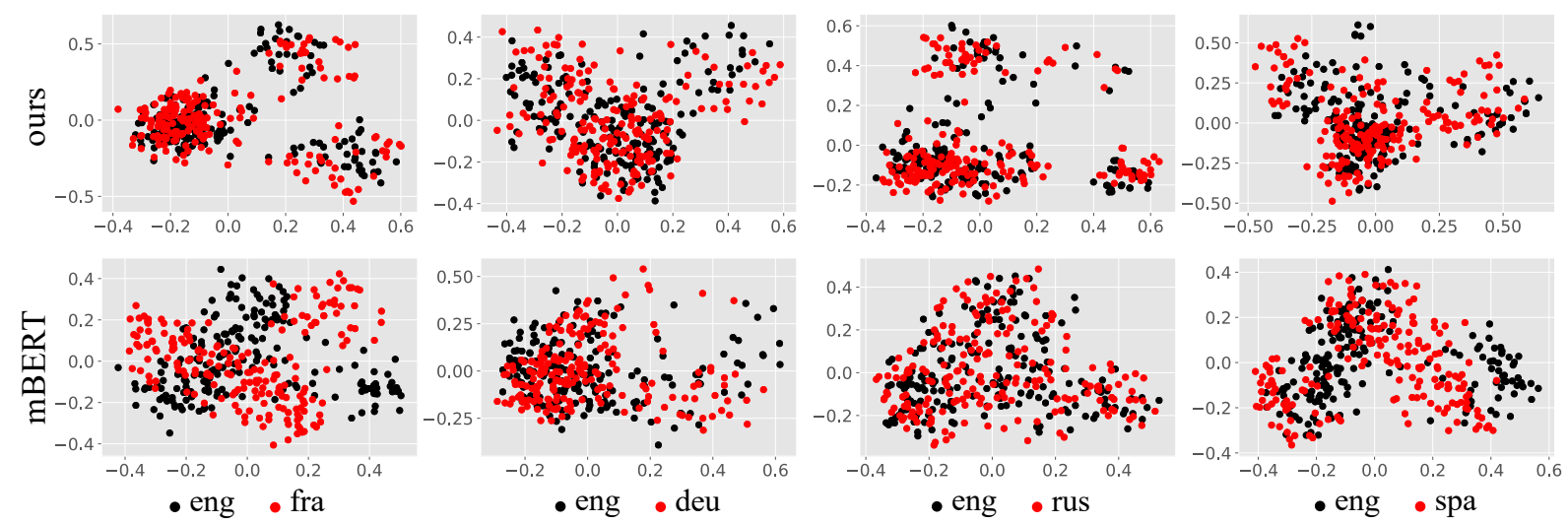

Figure 3: Visualizations of sentence embeddings of CMLM (first row) and mBERT (second row) in Tatoeba dataset in 2D. The target languages are all English and the source languages are French, German, Russian and Spanish.

We also discover that removing the first principal component of each monolingual space from sentence representations effectively eliminates the self language bias. Given a monolingual space $\boldsymbol{M}^{l_{1}} \in \mathbb{R}^{N \times d}$, where each row of $\boldsymbol{M}^{l_{1}}$ is a embedding in language $l_{1}$. For example, in the evaluation on Tatoeba dataset, the monolingual space matrix $M^{l_{1}}$ is computed with texts in language $l_{1}$ in Tatoeba. The principal component $c_{l_{1}}$ is the first right singular vector of $\boldsymbol{M}^{l_{1}}$. Given a representation $\boldsymbol{v}_{l_{1}}$ in language $l_{1}$, the projection of $\boldsymbol{v}_{l_{1}}$ onto $c_{l_{1}}$ is removed: $\hat{\boldsymbol{v}}_{l_{1}}=\boldsymbol{v}_{l_{1}}-\frac{\boldsymbol{v}_{l_{1}}^{T} c_{l_{1}}}{\left\|\boldsymbol{v}_{l_{1}}\right\|_{2}}$. The similarity score between $\boldsymbol{v}_{l_{1}}$ and $\boldsymbol{v}_{l_{2}}$ for cross-lingual retrieval is computed as: $\frac{\hat{\boldsymbol{v}}_{l_{1}}^{T} \hat{\boldsymbol{v}}_{l_{2}}}{\left\|\hat{\boldsymbol{v}}_{l_{1}}\right\|_{2}\left\|\hat{\boldsymbol{v}}_{l_{2}}\right\|_{2}}$.

As shown in the second and the fourth column in Fig. 2, with principal component removal (PCR), the language distribution of retrieved texts is much more uniform. We also explore PCR on the Tatoeba dataset. Table 8 shows the retrieval accuracy of multilingual model with and w/o PCR. PCR increases the overall retrieval performance for both models. This suggests the first principal components in each monolingual space primarily encodes language identification information.

We also visualize sentence embeddings on Tatoeba dataset in Fig. 3. Our model shows both weak and strong semantic alignment (Roy et al., 2020). Representations are close to others with similar semantics regardless of their languages (strong alignment), especially for French and Russian, where representations form several distinct clusters. Also representations from the same language tend to cluster (weak alignment). While representations from mBERT generally exhibit weak alignment.

\section{Conclusion}

We present a novel sentence representation learning method Conditional Masked Language Modeling (CMLM) for training on large scale unlabeled corpus. CMLM outperforms the previous stateof-the-art English sentence embeddings models, including those trained with (semi-)supervised signals. For multilingual representations, we discover that co-training CMLM with bitext retrieval and cross-lingual NLI finetuning achieves state-of-theart performance. We also find that multilingual representations have the same language bias and principal component removal can eliminate the bias by separating language identity information from semantics.

\section{Acknowledgments}

We would like to thank our teammates from Descartes, Google Brain and other Google groups for their feedback and suggestions. We also thank anonymous reviewers for their comments. Special thanks goes to Chen Chen and Hongkun Yu for help with TensorFlow model garden, and Arno Eigenwillig for help on releasing models on TensorFlow Hub.

\section{References}

Joshua Ainslie, Santiago Ontanon, Chris Alberti, Vaclav Cvicek, Zachary Fisher, Philip Pham, Anirudh Ravula, Sumit Sanghai, Qifan Wang, and Li Yang. 2020. ETC: Encoding long and structured inputs in transformers. In Proceedings of the 2020 Conference on Empirical Methods in Natural Language Processing (EMNLP), pages 268-284, Online. Association for Computational Linguistics. 
Mikel Artetxe and Holger Schwenk. 2019. Massively multilingual sentence embeddings for zeroshot cross-lingual transfer and beyond. Transactions of the Association for Computational Linguistics, 7:597-610.

Daniel Cer, Yinfei Yang, Sheng-yi Kong, Nan Hua, Nicole Limtiaco, Rhomni St John, Noah Constant, Mario Guajardo-Cespedes, Steve Yuan, Chris Tar, et al. 2018. Universal sentence encoder. arXiv preprint arXiv:1803.11175.

Muthu Chidambaram, Yinfei Yang, Daniel Cer, Steve Yuan, Yunhsuan Sung, Brian Strope, and Ray Kurzweil. 2019. Learning cross-lingual sentence representations via a multi-task dual-encoder model. In Proceedings of the 4th Workshop on Representation Learning for NLP (RepLANLP-2019), pages 250-259, Florence, Italy. Association for Computational Linguistics.

Alexis Conneau and Douwe Kiela. 2018. Senteval: An evaluation toolkit for universal sentence representations. In Proceedings of the Eleventh International Conference on Language Resources and Evaluation (LREC 2018).

Alexis Conneau, Douwe Kiela, Holger Schwenk, Loïc Barrault, and Antoine Bordes. 2017. Supervised learning of universal sentence representations from natural language inference data. In Proceedings of the 2017 Conference on Empirical Methods in Natural Language Processing, pages 670-680.

Jacob Devlin, Ming-Wei Chang, Kenton Lee, and Kristina Toutanova. 2019. Bert: Pre-training of deep bidirectional transformers for language understanding. In Proceedings of the 2019 Conference of the North American Chapter of the Association for Computational Linguistics: Human Language Technologies, Volume 1 (Long and Short Papers), pages 4171-4186.

Akiko Eriguchi, Melvin Johnson, Orhan Firat, Hideto Kazawa, and Wolfgang Macherey. 2018. Zeroshot cross-lingual classification using multilingual neural machine translation. arXiv preprint arXiv:1809.04686.

Fangxiaoyu Feng, Yinfei Yang, Daniel Cer, Naveen Arivazhagan, and Wei Wang. 2020. Languageagnostic bert sentence embedding. arXiv preprint arXiv:2007.01852.

Junjie $\mathrm{Hu}$, Sebastian Ruder, Aditya Siddhant, Graham Neubig, Orhan Firat, and Melvin Johnson. 2020. Xtreme: A massively multilingual multi-task benchmark for evaluating cross-lingual generalization. arXiv preprint arXiv:2003.11080.

Minqing $\mathrm{Hu}$ and Bing Liu. 2004. Mining and summarizing customer reviews. In Proceedings of the tenth ACM SIGKDD international conference on Knowledge discovery and data mining, pages 168-177.
Ryan Kiros, Yukun Zhu, Russ R Salakhutdinov, Richard Zemel, Raquel Urtasun, Antonio Torralba, and Sanja Fidler. 2015. Skip-thought vectors. In C. Cortes, N. D. Lawrence, D. D. Lee, M. Sugiyama, and R. Garnett, editors, Advances in Neural Information Processing Systems 28, pages 3294-3302. Curran Associates, Inc.

Zhenzhong Lan, Mingda Chen, Sebastian Goodman, Kevin Gimpel, Piyush Sharma, and Radu Soricut. 2020. Albert: A lite bert for self-supervised learning of language representations. In International Conference on Learning Representations.

Yinhan Liu, Myle Ott, Naman Goyal, Jingfei Du, Mandar Joshi, Danqi Chen, Omer Levy, Mike Lewis, Luke Zettlemoyer, and Veselin Stoyanov. 2019. Roberta: A robustly optimized bert pretraining approach. arXiv preprint arXiv:1907.11692.

Lajanugen Logeswaran and Honglak Lee. 2018. An efficient framework for learning sentence representations. In International Conference on Learning Representations.

Kumar Avinava Dubey Joshua Ainslie Chris Alberti Santiago Ontanon Philip Pham Anirudh Ravula Qifan Wang Li Yang Amr Ahmed Manzil Zaheer, Guru Guruganesh. 2020. Big bird: Transformers for longer sequences. In Advances in Neural Information Processing Systems. Curran Associates, Inc.

Marco Marelli, Stefano Menini, Marco Baroni, Luisa Bentivogli, Raffaella Bernardi, Roberto Zamparelli, et al. 2014. A sick cure for the evaluation of compositional distributional semantic models. In $L R E C$, pages 216-223.

Bo Pang and Lillian Lee. 2004. A sentimental education: Sentiment analysis using subjectivity summarization based on minimum cuts. In Proceedings of the 42nd Annual Meeting of the Association for Computational Linguistics (ACL-04), pages 271-278.

Bo Pang and Lillian Lee. 2005. Seeing stars: exploiting class relationships for sentiment categorization with respect to rating scales. In Proceedings of the 43rd Annual Meeting on Association for Computational Linguistics, pages 115-124.

Peter Prettenhofer and Benno Stein. 2010. Crosslanguage text classification using structural correspondence learning. In Proceedings of the 48th annual meeting of the association for computational linguistics, pages 1118-1127.

Colin Raffel, Noam Shazeer, Adam Roberts, Katherine Lee, Sharan Narang, Michael Matena, Yanqi Zhou, Wei Li, and Peter J. Liu. 2019. Exploring the limits of transfer learning with a unified text-to-text transformer. arXiv e-prints.

Nils Reimers and Iryna Gurevych. 2019. Sentencebert: Sentence embeddings using siamese bertnetworks. In Proceedings of the 2019 Conference on Empirical Methods in Natural Language Processing 
and the 9th International Joint Conference on Natural Language Processing (EMNLP-IJCNLP), pages 3973-3983.

Nils Reimers and Iryna Gurevych. 2020. Making monolingual sentence embeddings multilingual using knowledge distillation. In Proceedings of the 2020 Conference on Empirical Methods in Natural Language Processing (EMNLP), pages 4512-4525, Online. Association for Computational Linguistics.

Uma Roy, Noah Constant, Rami Al-Rfou, Aditya Barua, Aaron Phillips, and Yinfei Yang. 2020. Lareqa: Language-agnostic answer retrieval from a multilingual pool. arXiv preprint arXiv:2004.05484.

Sebastian Ruder, Anders Søgaard, and Ivan Vulić 2019. Unsupervised cross-lingual representation learning. In Proceedings of the 57th Annual Meeting of the Association for Computational Linguistics: Tutorial Abstracts, pages 31-38, Florence, Italy. Association for Computational Linguistics.

Richard Socher, Alex Perelygin, Jean Wu, Jason Chuang, Christopher D Manning, Andrew Y Ng, and Christopher Potts. 2013. Recursive deep models for semantic compositionality over a sentiment treebank. In Proceedings of the 2013 conference on empirical methods in natural language processing, pages 1631-1642.

Sandeep Subramanian, Adam Trischler, Yoshua Bengio, and Christopher J Pal. 2018. Learning general purpose distributed sentence representations via large scale multi-task learning. In International Conference on Learning Representations.

Ellen M Voorhees and Dawn M Tice. 2000. Building a question answering test collection. In Proceedings of the 23rd annual international ACM SIGIR conference on Research and development in information retrieval, pages 200-207.

Adina Williams, Nikita Nangia, and Samuel Bowman. 2018. A broad-coverage challenge corpus for sentence understanding through inference. In Proceedings of the 2018 Conference of the North American Chapter of the Association for Computational Linguistics: Human Language Technologies, Volume 1 (Long Papers), pages 1112-1122.

Yinfei Yang, Daniel Cer, Amin Ahmad, Mandy Guo, Jax Law, Noah Constant, Gustavo Hernandez Abrego, Steve Yuan, Chris Tar, Yun-Hsuan Sung, et al. 2019a. Multilingual universal sentence encoder for semantic retrieval. arXiv preprint arXiv:1907.04307.

Yinfei Yang, Gustavo Hernandez Abrego, Steve Yuan, Mandy Guo, Qinlan Shen, Daniel Cer, Yun-hsuan Sung, Brian Strope, and Ray Kurzweil. 2019b. Improving multilingual sentence embedding using bidirectional dual encoder with additive margin softmax. In Proceedings of the Twenty-Eighth International Joint Conference on Artificial Intelligence, IJCAI-19, pages 5370-5378. International
Joint Conferences on Artificial Intelligence Organization.

Yinfei Yang, Yuan Zhang, Chris Tar, and Jason Baldridge. 2019c. Paws-x: A cross-lingual adversarial dataset for paraphrase identification. In Proceedings of the 2019 Conference on Empirical Methods in Natural Language Processing and the 9th International Joint Conference on Natural Language Processing (EMNLP-IJCNLP), pages 3678-3683.

Zhilin Yang, Zihang Dai, Yiming Yang, Jaime Carbonell, Russ R Salakhutdinov, and Quoc V Le. 2019d. Xlnet: Generalized autoregressive pretraining for language understanding. Advances in Neural Information Processing Systems, 32:5753-5763.

Ziyi Yang, Chenguang Zhu, and Weizhu Chen. 2019e. Parameter-free sentence embedding via orthogonal basis. In Proceedings of the 2019 Conference on Empirical Methods in Natural Language Processing and the 9th International Joint Conference on Natural Language Processing (EMNLP-IJCNLP), pages 638-648.

Yang You, Jing Li, Sashank Reddi, Jonathan Hseu, Sanjiv Kumar, Srinadh Bhojanapalli, Xiaodan Song, James Demmel, Kurt Keutzer, and Cho-Jui Hsieh. 2020. Large batch optimization for deep learning: Training bert in 76 minutes. In International Conference on Learning Representations. 


\section{A Methods for Representations}

We evaluate different representations method in Transformer-base models, including CMLM and BERT base (using the model on official Tensorflow Hub). The experiments are conducted on SentEval. Results in Table 9 show that MEAN representation exhibit better performance than CLS and MAX representations.

\section{B Experiments with different Masking ratios}

We test with different masking ratios in CMLM training data. Specifically, We tried masking 40 , 60, 80 and 100 tokens of 256 tokens in the CMLM data. Performance of obtained models on SentEval are presented in Appendix B.

\section{Training Configurations and Implementation Details}

Projection $P$ in the CMLM modeling. Let $\mathrm{h}$ denote the dimension of the input sentence vector (e.g. $\mathrm{h}=768$ in BERT base; $\mathrm{h}=1024$ in BERT large). Let $F C\left(h_{1}, h_{2}, n\right)$ denote a fully connected layer with input dimension $h_{1}$, output dimension $h_{2}$ and nonlinearity function $n$. The three layers are $F C(h, 2 \times h, \operatorname{ReLU}), F C(2 \times h, 2 \times h, \mathrm{ReLU})$, $F C(2 \times h, h$, None $)$. We tried projections without intermediate layers and observed a drop in training LM accuracy. Adding more layers doesn't improve the MLM accuracy or downstream tasks performance. Using $2 \times h$ is empirically chosen based on preliminary experiments. Other hidden sizes are also explored.

Configurations for multilingual representations learning. In general, larger batch sizes improve performance until we reach 2048 , since each example will see more "mismatched" examples. After 2048, we do not see obvious improvements in performance from increasing batch size. We'll add detailed results on this in the final version. The training steps for different stages are decided on a validation set.

Training Data and infrastructure. English pretraining takes 5 days on 64 TPUs using 1TB of data from Common Crawl dumps 2020-1, 202005, 2020-10. More data could be beneficial, but would increase training time.

\section{Reliability of XEVAL}

In this section, we want to discuss the reliability of XEVAL. XEVAL contains sentence-level data and we expect its translation not to be too challenging. Inspection by in-house bilingual speakers also confirms the high quality of translation. Human translation is always preferred but we are limited by budget and annotator resources (especially for low-resource languages).

\section{E CMLM's Comparison with Next Sentence Prediction (NSP) and Potential Limitations.}

We tried MLM (CC) with and w/o NSP and it does not make much difference on SentEval. Training NSP accuracy quickly converge to $95 \%$, indicating that NSP is not a challenging task.

Sentence embedding methods like CMLM can be less effective for sequence labeling (e.g., NER) and natural language generation (NLG) and question answering (Q\&A).

\section{F Performance Variances}

We provide the performance variances of CMLM base and CMLM large on SentEval dataset in Table 11 . 


\begin{tabular}{l|cccccccccc}
\hline Model & MR & CR & SUBJ & MPQA & SST & TREC & MRPC & SICK-E & SICK-R & Avg. \\
\hline CMLM MAX & 82.8 & 88.9 & 96.2 & 89.2 & 87.81 & 89.8 & 72.1 & 82.1 & 83.7 & 85.8 \\
CMLM MEAN & 83.6 & 89.9 & 96.2 & 89.3 & 88.5 & 91.0 & 69.7 & 82.3 & 83.4 & $\mathbf{8 6 . 0}$ \\
CMLM CLS & 79.1 & 84.3 & 94.2 & 86.9 & 84.9 & 82.6 & 68.4 & 79.3 & 81.7 & 82.4 \\
\hline BERT base MAX & 79.6 & 85.5 & 94.6 & 87.3 & 83.0 & 90.0 & 65.6 & 75.5 & 78.1 & 82.1 \\
BERT base MEAN & 81.6 & 87.4 & 95.2 & 87.8 & 85.8 & 90.6 & 71.1 & 79.3 & 80.5 & $\mathbf{8 4 . 3}$ \\
BERT base CLS & 79.9 & 83.9 & 93.8 & 85.4 & 86.1 & 81.0 & 69.5 & 62.5 & 48.8 & 76.8 \\
\hline
\end{tabular}

Table 9: Performance of sentence representations model with different representations method (MAX, MEAN and CLS).

\begin{tabular}{c|cccccccccc}
\hline Mask Tokens & MR & CR & SUBJ & MPQA & SST & TREC & MRPC & SICK-E & SICK-R & Avg. \\
\hline 40 & 81.8 & 89.3 & 95.3 & 87.8 & 87.0 & 90.2 & 68.5 & 77.5 & 77.6 & 83.9 \\
60 & $\mathbf{8 3 . 7}$ & 89.5 & 95.8 & 88.9 & 88.0 & 90.3 & 68.7 & 79.5 & 82.8 & 85.4 \\
$\mathbf{8 0}$ & 83.6 & $\mathbf{8 9 . 9}$ & $\mathbf{9 6 . 2}$ & $\mathbf{8 9 . 3}$ & $\mathbf{8 8 . 5}$ & $\mathbf{9 1 . 0}$ & 69.7 & $\mathbf{8 2 . 3}$ & $\mathbf{8 3 . 4}$ & $\mathbf{8 6 . 0}$ \\
100 & 83.2 & 89.5 & 95.5 & 88.7 & 88.0 & 90.8 & $\mathbf{7 0 . 0}$ & 81.5 & 82.7 & 85.6 \\
\hline
\end{tabular}

Table 10: Performance with different masking ratios in data (X-out-of-256) of CMLM base on SentEval.

\begin{tabular}{l|ccccccccc}
\hline Model & MR & CR & SUBJ & MPQA & SST & TREC & MRPC & SICK-E & SICK-R \\
\hline CMLM base & $83.6 \pm 0.2$ & $89.9 \pm 0.4$ & $96.2 \pm 0.1$ & $89.3 \pm 0.3$ & $88.5 \pm 0.2$ & $91.0 \pm 0.8$ & $69.7 \pm 0.6$ & $82.3 \pm 0.3$ & $83.4 \pm 0.4$ \\
CMLM large & $85.6 \pm 0.2$ & $89.1 \pm 0.3$ & $96.6 \pm 0.2$ & $89.3 \pm 0.3$ & $91.4 \pm 0.1$ & $92.4 \pm 0.7$ & $70.0 \pm 1.0$ & $82.2 \pm 0.5$ & $84.5 \pm 0.4$ \\
\hline
\end{tabular}

Table 11: Performance variances of CMLM base and CMLM large on SentEval. 